\title{
Analysis of High Frequency Planar Sandwich Transformers for Switching Converters
}

\author{
Jun W. Lu \\ School of Microelectronic Engineering, Faculty of SCT, \\ Griffith University, QLD 4111, AUSTRALIA \\ Francis P Dawson* \\ * Dept. of Electrical \& Computer Engineering, University of Toronto, \\ Toronto, Ontorio, M5S 1A4, CANADA \\ Sotoshi Yamada** \\ **Lab. of Magnetic Field Control and Applications, Faculty of Technology, \\ Kanazawa University, Kanazawa 920, JAPAN
}

\begin{abstract}
This paper presents the results of investigations on a planar sandwich air-gapped transformer with two different winding configurations. Experimental results of the coil self inductances, voltage ratio versus frequency, and the numerical results for the magnetic flux and eddy-current distributions are presented. It is shown that the voltage ratio and turns ratio are not equivalent and that the voltage ratio is frequency dependent.
\end{abstract}

\section{INTRODUCTION}

Various planar magnetic components have been developed recently in order to address the disadvantages of conventional magnetic structures. Advantages of planar designs include a low profile, good heat transfer properties, and operation at high power densities because of low power losses. These advantages are important if one is to realise a reduction in the size of magnetic components in high frequency converters [1]. Also, lack of accurate data on parasitic elements, such as leakage inductance, winding capacitance, and skin and proximity effect makes it very difficult to design high-frequency magnetic devices correctly the first time. Several planar inductive components with different configurations have been reported, for instance the Sandwich Transformer [2], the Matrix Transformer [3] and the Thin Film Microtransformer with Circular Spiral Coils [4].

This paper presents the results of investigations on two types of planar sandwich air-gapped transformer designs. The first step is to characterize the circuit model as a function of frequency. The assumptions, considered include a linear material and no hysterisis effects. It is therefore not necessary to characterize the nature of the input voltage. The next step is to consider the flux distribution within the coil so as to assist us in determining how effectively the core volume is being utilized and how uniform the flux distribution is. Knowledge of the eddy current distribution allows us to calculate the copper losses and the eddy current losses within the magnetic material. A TDK (Mn$\mathrm{Zn}$ ) high-frequency planar magnetic material is used to investigate the inductance and voltage ratio versus frequency characteristics and also the distributions of magnetic flux and eddy-currents for two different winding configurations. A CAD/CAE software package which employs the boundary element method (BEM) has been used to obtain the numerical results.

\section{Planar SANDWICH STRUCTURE AND COMPUTATION MODEL}

DC-DC converters operating at high frequencies near $1 \mathrm{MHz}$ require inductance values in the range of a few micro-henries. Sandwich magnetic designs with open and closed structures and with a spiral configuration are most commonly used in high frequency applications. In our experiments, the primary and secondary coils have the same number of turns. Type I has three turns and type II has one turn. The dimensions of the ferrite core are $17.6 \times 17.6 \times 2.0 \mathrm{~mm}$.
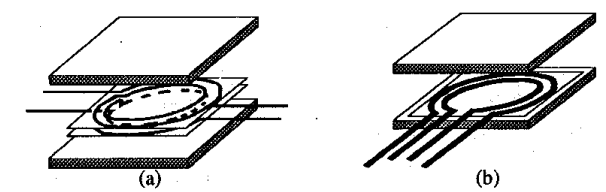

Fig. 1 Planar sandwich spiral-type winding configurations, (a) type I magnetic and winding structure, (b) type II magnetic and winding structure.

The basic open core magnetic structure with a spiral winding configurations is shown in Fig. 1. A two dimensional axi-symmetric unbounded structure is assumed for computational purposes and is illustrated in Fig. 2 . The material properties for the ferrite material, insulator and coil, such as permeability, permittivity and conductivity are considered in the calculation. A PC-based CAD/CAE tool 
(OERSTED 2D/RS) which employs the boundary element method (BEM) has been used to obtain the numerical results.

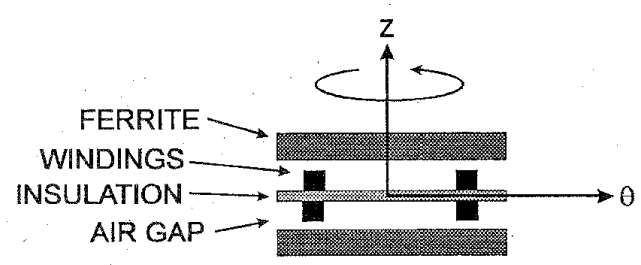

Fig 2. computation model calculated by BEM

\section{INDUCTANCE vS FREQUENCY CHARACTERISTICS}

Figure 3 presents the experimental results for the inductance vs frequency characteristics of a planar magnetic device with a spiral winding configuration. A HP $4285 \mathrm{~A}$ precision LCR meter was used to obtain the results over a frequency range of $100 \mathrm{KHz}$ to $1 \mathrm{MHz}$. The inductance $\mathrm{L}$ decreases with increasing frequency due to the decrease in material permeability with increasing frequency.

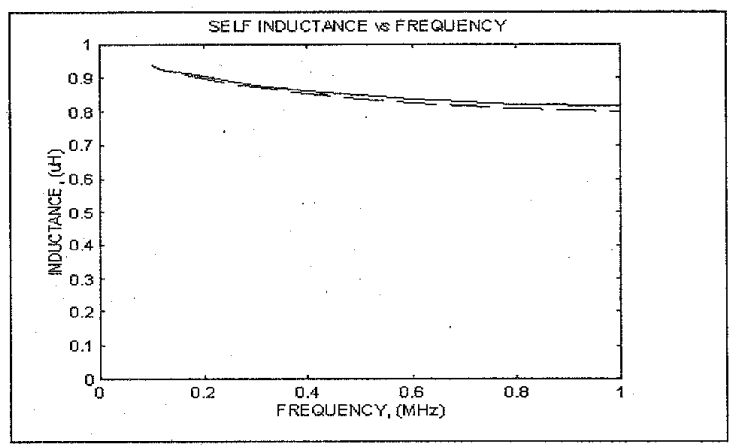

(a) type I magnetic and winding structure

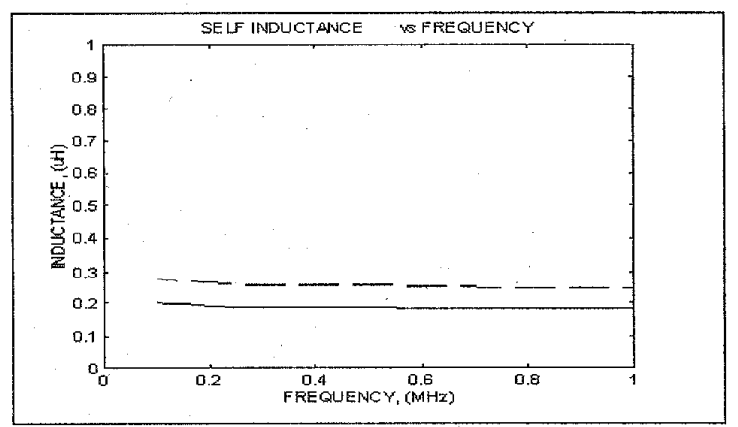

(b) type II magnetic and winding structure

Fig. 3 Self inductance vs frequency characteristics, where - and indicate the results for primary and secondary windings respectively.

\section{Yoltage Ratio vs FREQUENCY CHARACTERISTICS}

The input and output voltage ratio versus frequency characteristics for the no-load case is investigated. The voltage ratio for opened planar magnetic core structures shown in Fig. 4 has a frequency dependent characteristic. The result is similar to the results which have been obtained for the thin film microtransformer with closed magnetic core structure [4]. The measured results also indicate that the voltage ratio remains relatively constant for type I transformer but has a peak at $5 \mathrm{MHz}$ for type II transformer. Magnetic coupling is rather small at low frequencies and thus the voltage ratio is not equal to the winding ratio that is $\mathrm{N}_{\text {out }} / \mathrm{N}_{\text {in }} \neq \mathrm{V}_{\text {out }} / \mathrm{V}_{\text {in }}$.

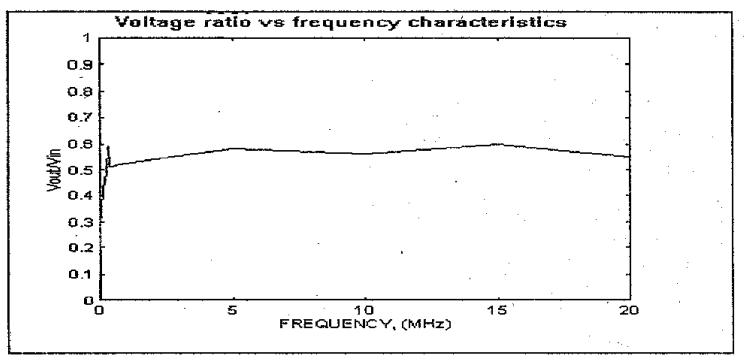

(a) type I magnetic and winding structure

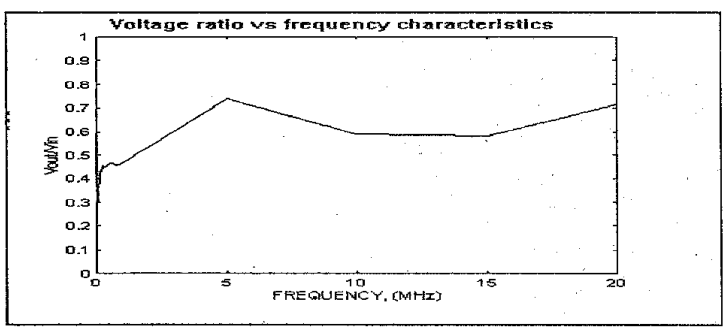

(b) type II magnetic and winding structure

Fig. 4 Voltage ratio versus frequency characteristics

\section{MAgnetic Flux Distribution At $1 \mathrm{MHz}$}

The time harmonic magnetic field distribution within the core and winding was calculated. A large number of boundary elements are considered for the inside area of the structure, particularly around the coil area. Fig.5 shows the computed results for the magnetic flux under the condition that the primary winding is excited and the secondary winding is open circuited. The numerical results show that the distribution of magnetic flux in the planar magnetic open core structures is different compared to conventional magnetic structures. The flux is shaped by the induced eddy-current in the secondary coil. Fig. 5 (a) shows that the magnetic flux leaks out of the ferrite. This leakage field 
may generate EMI and could pose problems. In contrast, Fig. 5 (b) shows no signs of an external leakage field.

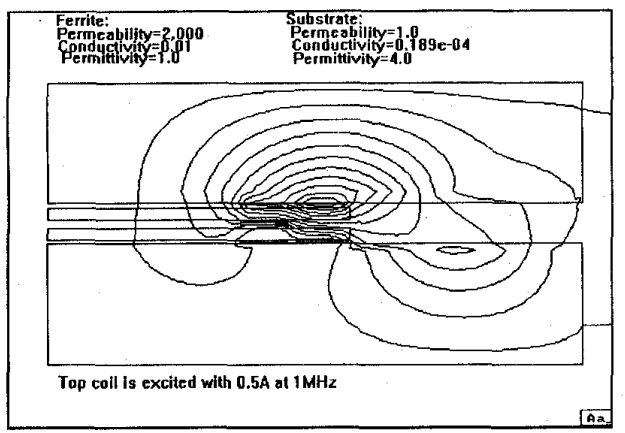

(a) axi-symmetric cross section of type I magnetic and winding structure

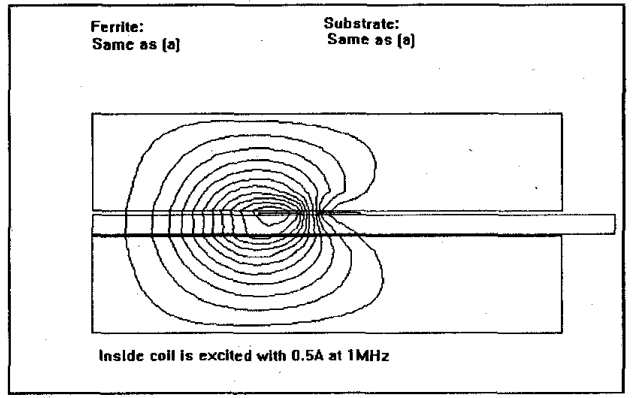

(b) axi-symmetric cross section of type II magnetic and winding structure

Fig. 5 Distributions of magnetic flux in planar spiral-type transformer

\section{EDDY-CURRENT DISTRIBUTION AT $1 \mathrm{MHz}$}

Eddy-current loss is a serious problem in both windings and magnetic cores at high frequencies. The proximity effect is often more important than skin effect in planar magnetic structures. Fig.6 shows the

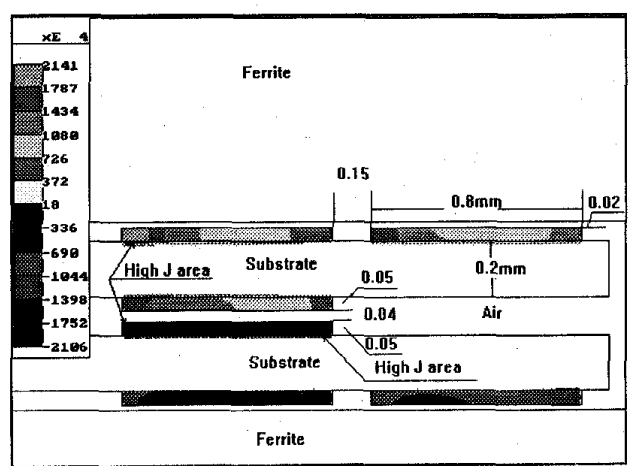

(a) eddy-current distribution in axi-symmetric cross section of type II magnetic and winding structure.

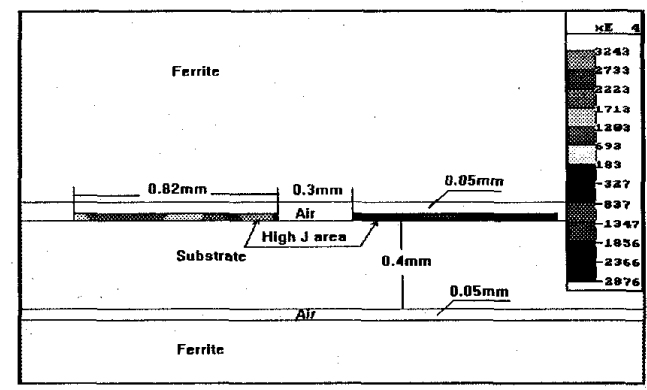

(b) eddy-current distribution in axi-symmetric cross section of type II magnetic and winding structure

Fig. 6 Induced eddy-currents in planar spiral-type transformers

numerical results. The excitation current is applied to the primary winding and the secondary winding is open circuited in the calculation. From this figure it is evident that the eddy-current distribution is sensitive to the nature of the winding configuration.

\section{CONCLUSIONS AND FUTURE WORK}

The paper presented experimental and numerical results for two planar air gapped high frequency transformers. The primary coil was subjected to a $100 \mathrm{kHz}$ to $1 \mathrm{MHz}$ sinusoidal current source and the secondary coil was left open circuited. The inequality between the secondary to primary voltage ratio and the turns ratio indicates that the magnetic coupling is quite small. The numerical results show that the distribution of magnetic flux and eddy-currents in the planar magnetic open core structures is different compared to conventional magnetic structures. The numerical results have been obtained using a CAD/CAE tool which employs the boundary element method. Future work will concentrate on optimizing the design of the magnetic system subject to constraints such as a desired magnetic coupling ratio, desired heat transfer materials, permissible values of EMI and the calculation of losses which include hysterisis effects.

\section{REFERENCES}

[1] Richard Farrington, Milan M Jovanovic, and Fred C Lee, "Design Oriented Analysis of Reactive Power in Resonant Converters", IEEE TRANS. ON POWER ELECTRONICS, Vol .8, No.4, pp. 411-422, Oct. 1993.

[2] J. G. Kassakian, M. F. Schlecht, "High-Frequency High Density Converters for Distributed Power Supply Systems", Proceedings of the IEEE, Vol. 76, No. 4, pp. 362-376, April 1988.

[3] Khai D. Ngo, E. Alpizar and J. Kenneth Watson, "Modeling of Magnetizing Inductance and Leakage Inductance in a Matrix Transfomer; IEEE TRANS. ON POWER ELECTRONICS, Vol .8, No.2, pp 200-207, April 1993.

[4] Kazuyuki Yanaguchi, Shigehiro Ohnuma, etc., "Characteristics of a Thin Film Microtransformer with Circular Spiral Coils" IEEE TRANS. ON MAGNETICS, Vol. 29, No.5, pp 2232-2237, Sep. 1993. 